\title{
Article \\ Evaluation of the Simultaneous Operation of the Mechanisms for Cross-Border Interchange and Activation of the Regulating Reserves
}

\author{
Marcel Topler *(i) and Boštjan Polajžer (D)
}

Citation: Topler, M.; Polajžer, B. Evaluation of the Simultaneous Operation of the Mechanisms for Cross-Border Interchange and Activation of the Regulating Reserves. Appl. Sci. 2021, 11, 8188. https:// doi.org/10.3390/app11178188

Academic Editors: Federico Barrero and Mario Bermúdez

Received: 11 August 2021

Accepted: 1 September 2021

Published: 3 September 2021

Publisher's Note: MDPI stays neutral with regard to jurisdictional claims in published maps and institutional affiliations.

Copyright: (c) 2021 by the authors. Licensee MDPI, Basel, Switzerland. This article is an open access article distributed under the terms and conditions of the Creative Commons Attribution (CC BY) license (https:// creativecommons.org/licenses/by/ $4.0 /)$.
Faculty of Electrical Engineering and Computer Science, University of Maribor, SI-2000 Maribor, Slovenia; bostjan.polajzer@um.si

* Correspondence: marcel.topler@um.si

\begin{abstract}
This article examines the mechanisms for cross-border interchange of the regulating reserves (RRs), i.e., the imbalance-netting process (INP) and the cross-border activation of the RRs (CBRR). Both mechanisms are an additional service of frequency restoration reserves in the power system and connect different control areas (CAs) via virtual tie-lines to release RRs and reduce balancing energy. The primary objective of the INP is to net the demand for RRs between the cooperating CAs with different signs of interchange power variation. In contrast, the primary objective of the CBRR is to activate the RRs in the cooperating CAs with matching signs of interchange power variation. In this way, the ancillary services market and the European balancing system should be improved. However, both the INP and CBRR include a frequency term and thus impact the frequency response of the cooperating CAs. Therefore, the impact of the simultaneous operation of the INP and CBRR on the load-frequency control (LFC) and performance is comprehensively evaluated with dynamic simulations of a three-CA testing system, which no previous studies investigated before. In addition, a function for correction power adjustment is proposed to prevent the undesirable simultaneous activation of the INP and CBRR. In this way, area control error (ACE) and scheduled control power are decreased since undesired correction is prevented. The dynamic simulations confirmed that the simultaneous operation of the INP and CBRR reduced the balancing energy and decreased the unintended exchange of energy. Consequently, the LFC and performance were improved in this way. However, the impact of the INP and CBRR on the frequency quality has no unambiguous conclusions.
\end{abstract}

Keywords: frequency quality; load-frequency control; regulating reserves; cross-border interchange; cross-border activation

\section{Introduction}

\subsection{Motivation and Literature Review}

The mechanisms for cross-border interchange and activation of the regulating reserves (RRs) are evolving due to the expensive balancing energy, and are included in the European Union's current regulations [1,2]. They are put in operation in continental Europe by the members of the European network of transmission system operators for electricity (ENTSOE) [3]. Since the first implementation of the cross-border interchange of the RRs, i.e., the imbalance-netting process (INP), in 2008, the cumulative value of all the netted imbalances amounted to more than $€ 600$ million by the third quarter of 2020 [4]. The total monthly volume of netted imbalances for September 2020 was 698.69 GWh, which amounts to $€ 13.38$ million. Moreover, the monthly avoided positive and negative RRs activations amount to a minimum of $10 \%$ to as much as $85 \%$. The further development of the INP with the functionality of the cross-border activation of the RRs (CBRR), will additionally reduce the activation of the RRs and increase the associated savings. 
To avert the activation of the RRs with different signs in the cooperating CAs, and thus reduce the RRs, a grid-control cooperation (GCC) platform was implemented in Germany, where four transmission system operators (TSOs), i.e., 50 Hertz, Amprion, TenneT, and TransnetBW have been collaborating since 2008 [5]. In the following years, many continental European countries joined and the GCC platform developed into the international GCC (IGCC) platform, with the aim to further reduce RRs and increase the reliability of the power system's operation [6-9]. Hence, the INP was developed and put in operation, where the cooperating CAs with different signs of power variations can exchange the balancing energy and thus compensate the power variations $[10,11]$. Therefore, CAs with a surplus of power can exchange with CAs having power shortages [12]. A comparable approach, i.e., area control error (ACE) diversity interchange, was implemented in North America in 1993, but does not consist of actual responses from the control units [13-15].

A further reduction of power-system operating costs and increasingly stringent requirements for the quality of the Load-Frequency Control (LFC) defined by the new network codes require further development of the INP with a functionality that will enable CBRR [16]. Therefore, in the first quarter of 2020, the development of the CBRR started that will be put in operation in continental Europe in 2022 [3]. The aim of the development and operation of the CBRR is to improve the ancillary services market and the European balancing system [17]. Similar to the INP, the same control-demand approach and implementation in the control structure will be used for the CBRR. However, the primary objective of the CBRR will be the activation of the RRs in the cooperating CAs and importing into its own $\mathrm{CA}$, thereby reducing the balancing energy [18]. CBRR will only be achievable if the cooperating CAs have matching signs of power deviations. Consequently, CAs with a surplus of power can activate the RRs only in CAs with a surplus of power. Both mechanisms, i.e., INP and CBRR, reduce the balancing energy, while releasing the RRs and, therefore, reducing the associated economic costs. This increases the economic benefits, as the energy exchanged by the INP and activated by the CBRR is additionally financially compensated [19].

A basic schematic diagram of the LFC, INP, and CBRR is given in Figure 1 (left), where the order of the operation is clearly seen. Note here that the correction power is the output of the INP I CBRR block. The main distinction among the INP and the CBRR is in the requirements to compensate for the imbalances among the cooperating CAs. The aim of the INP is to avert the simultaneous activation of RRs with different signs in cooperating CAs, i.e., to net the demand for balancing energy between CAs with different signs of demand power. In contrast, the aim of CBRR is to activate the demand for balancing energy in cooperating CAs with matching signs of demand power. The INP and the CBRR link all the CAs to a joint portal of virtual tie-lines where the INP or CBRR optimization is performed. Note that a virtual tie-line means an additional input of the controllers of the cooperating CAs that has the same effect as a measuring value of a physical interconnector and allows exchange of electric energy between the cooperating CAs [1]. The main objectives of the INP optimization are given in $[20,21]$, whereas the main objectives of the CBRR optimization are given in $[22,23]$.
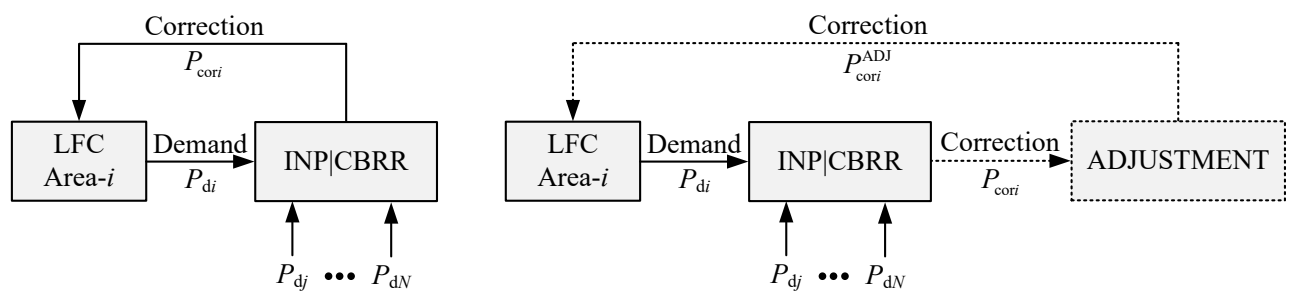

Figure 1. Schematic diagram of the LFC, INP, CBRR (left) and the function for correction power adjustment (right).

There has been a surge in the application of machine learning and statistical framework to solve similar problems focused in this paper. The authors in [24] explore the influencing 
factors of consumer purchase intention of cross-border e-commerce based on a wireless network and machine learning in order to provide decision support for the operation of e-commerce and to promote the better development of cross-border e-commerce. Several model-based experimental design techniques have been developed for design in domains with partial available data about the underlying process. The authors in [25] focus on a powerful class of model-based experimental design called the mean objective cost of uncertainty. To achieve a scalable objective-based experimental design, a graph-based mean objective cost of uncertainty with Bayesian optimization framework is proposed. A thorough review of the issues of data localization and data residency is given in [26], in addition to clarifying cross-border data flow restrictions and the impact of cross-border data flows in Asia.

\subsection{Contribution and Structure of the Paper}

Generally, the INP and the CBRR should have a positive impact on the LFC and performance. However, the quality of the frequency is continually decreasing [27]. Therefore, the impact of the INP and the CBRR on the frequency quality, the LFC, and performance in a three-CA test system was examined separately in $[20,22]$ with dynamic simulations. In [21], the impact of INP on power-system dynamics is shown and an eigenvalue analysis of a two-CA system is conducted. The impact of CBRR on the power-system dynamics is shown in [23], and a modified implementation of the CBRR is proposed that has no impact on the system's eigendynamics. This article extends these earlier results with an in-depth evaluation of the simultaneous operation of the mechanisms for cross-border interchange and activation of the RRs, which was not studied before. Dynamic simulations are performed for all the cases where the simultaneous operation of the INP and the CBRR is possible. In addition, a function for correction-power adjustment is proposed as one of the contributions of this paper, since small delays in demand power sign change could cause undesired simultaneous activation of the INP and the CBRR. In this way, ACE and scheduled control power are decreased, since udesired correction is prevented. A basic schematic diagram of the LFC, INP, and CBRR and the function for correction power adjustment is given in Figure 1 (right), where the order of operation is clearly seen. Note here that the correction power is the output of the adjustment block. As far as we know, no researchers have examined the impact of the simultaneous operation of the INP and CBRR on the LFC and performance.

This article consists of the following parts: In Section 2, the elemental concepts of the LFC, the INP and the CBRR are described. Simultaneous operation of the INP and the CBRR is also described. Additionally, a function for correction power adjustment is proposed as one of the main contributions of this article, which prevents undesired correction. Section 3 describes indicators for evaluation of the frequency quality, LFC and performance, rate of change of frequency (RoCoF), balancing energy, unintended exchange of energy and energy exchange. In Section 4 , a three-CA test system with the INP and the CBRR is described. Two types of test cases were performed with the dynamic simulations, i.e., step change of the load and the random load variation. The primary contribution of this article is given in Section 5, where the impact is given of the simultaneous operation of the INP and the CBRR on the frequency quality, the LFC, and performance. Lastly, Section 6 outlines the main conclusions and outlines future work.

\section{LFC, INP, and CBRR}

\section{1. $L F C$}

An interconnected power system consists of a large number of CAs that are connected via tie-lines. Each individual TSO maintains the frequency of each CA within predefined standard limits and the tie-line power flows with neighboring CAs within prespecified tolerances, which are the main objectives of the LFC [28]. This is generally accomplished by reducing the $\mathrm{ACE}$, which is, for the $\mathrm{CA} i$, defined as 


$$
A C E_{i}=\Delta P_{i}+B_{i} \Delta f_{i}
$$

where $\Delta P_{i}=\left(P_{\mathrm{a} i}-P_{\mathrm{s} i}\right)$ and $\Delta f_{i}=\left(f_{\mathrm{a} i}-f_{\mathrm{s} i}\right)$ denote interchange power variation and frequency deviation, respectively. Note that $P_{\mathrm{a} i}$ and $f_{\mathrm{a} i}$ denote actual values, while $P_{\mathrm{s} i}$ and $f_{\mathrm{s} i}$ denote scheduled values. Furthermore, $B_{i}$ is the frequency-bias coefficient [1]. Note that $A C E_{i}>0$ denotes that the generation is higher than the load; hence, the CA is denoted as "long". Similarly, a CA is denoted as "short" when $A C E_{i}<0$.

The basic LFC framework of the CA $i$ is shown in Figure 2, where LPF denotes a low pass filter and $\mathrm{SH}$ a sample and hold, with the value of a sampling time $T_{\mathrm{s}}=2 \mathrm{~s}$. A negative control-feedback is characterized as -1 gain and PI denotes a proportionalintegral controller. Scheduled control power $\Delta P_{\mathrm{sc} i}$ denotes the output of LFC, which is appointed to the participating control units that change the electrical control power $\Delta P_{\mathrm{e} i}$ appropriately. Neglecting the losses, then $\Delta P_{\mathrm{e} i}$ is, for the CA $i$, defined as

$$
\Delta P_{\mathrm{e} i}=\Delta P_{\mathrm{L} i}+\Delta P_{i}
$$

where $\Delta P_{\mathrm{L} i}$ denotes the load-power variation. Note that $\Delta P_{\mathrm{e} i}$ is well-known as the balancing energy, whereas, instead of LFC reserve, the term RR is generally used.

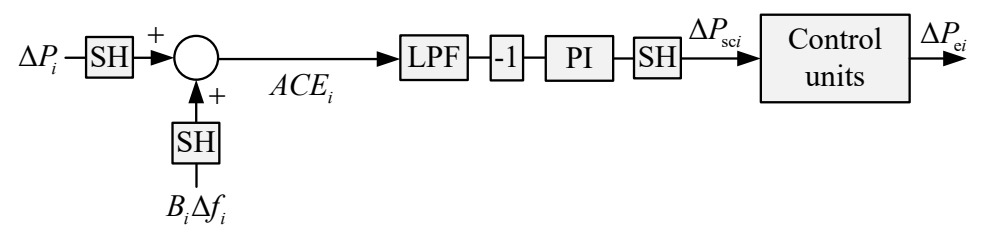

Figure 2. Schematic block diagram representation of the LFC in the CA $i$.

\subsection{INP}

The basic principle of the INP operation and a steady-state correction value calculation with the INP for three CAs is given in [20]. A control-demand concept is used for the INP and a schematic block diagram representation is shown in Figure 3. Commonly, N CAs can be connected via virtual tie-lines, i.e., they can all activate the INP via the interchange factors $K_{i}^{\mathrm{INP}}, K_{j}^{\mathrm{INP}}, \ldots, K_{N}^{\mathrm{INP}}$ marked with the green rectangle. Note that the factor $K_{j}^{\mathrm{INP}}$ represents the size of the INP interchange of the $j$-th CA in the $i$-th CA, where $K_{j}^{\mathrm{INP}}=0$ determines that the possible INP interchange is equal to $0 \%$, whereas $K_{j}^{\mathrm{INP}}=1$ determines that the possible INP interchange is equal to $100 \%$.

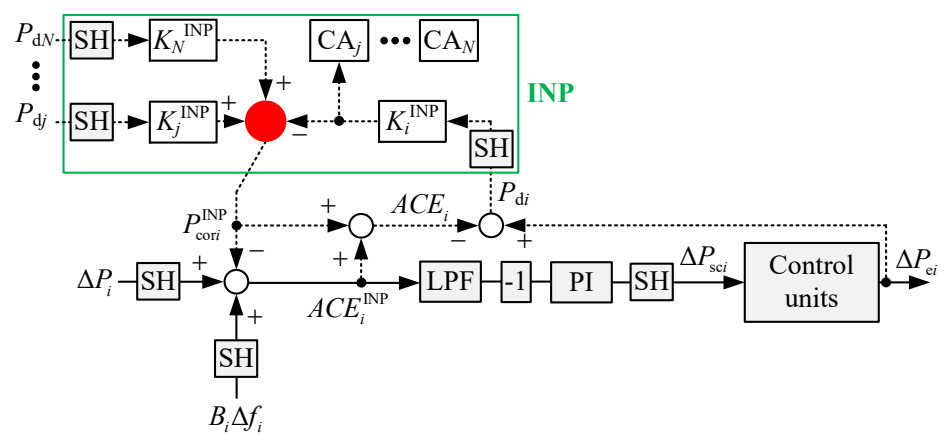

Figure 3. Schematic block diagram representation of the LFC in the CA $i$ (solid line) with the INP (solid and dotted line).

Moreover, the values of $K_{i}^{\mathrm{INP}}, K_{j}^{\mathrm{INP}}, \ldots, K_{N}^{\mathrm{INP}}$ can be different. The cooperating CAs are connected to the "red" summator, forming virtual tie-lines, as shown in Figure 3. The input variables to the "red" summator are the demand powers of the cooperating CAs, i.e., $P_{\mathrm{d} i}$, 
$P_{\mathrm{d} j}, \ldots, P_{\mathrm{d} N}$. The demand power of the CA $i$ characterizes the maximum interchange power for the CA $i$ among the cooperating $\mathrm{CAs}$ and is defined as

$$
P_{\mathrm{d} i}=\Delta P_{\mathrm{e} i}-A C E_{i}
$$

according to $[3,29]$.

Introducing (1) and (2) in (3) gives the following relation:

$$
P_{\mathrm{d} i}=\Delta P_{\mathrm{L} i}-B_{i} \Delta f_{i}
$$

The power imbalance between generation and load in addition to $K_{i}^{\mathrm{INP}} P_{\mathrm{d} i}$ from the $\mathrm{CA} i, K_{j}^{\mathrm{INP}} P_{\mathrm{d} j}$ from CA $j, \ldots, K_{N}^{\mathrm{INP}} P_{\mathrm{dN}}$ from CA $N$ is, for the $\mathrm{CA} i$, defined as

$$
A C E_{i}^{\mathrm{INP}}=B_{i} \Delta f_{i}+\Delta P_{i}+K_{i}^{\mathrm{INP}} P_{\mathrm{d} i}-\left(K_{j}^{\mathrm{INP}} P_{\mathrm{d} j}+, \ldots,+K_{N}^{\mathrm{INP}} P_{\mathrm{d} N}\right) .
$$

The output variables of the "red" summator are the correction powers of the cooperating CAs, i.e., $P_{\text {cori } i}^{\mathrm{INP}}, P_{\text {cor } j}^{\mathrm{INP}}, \ldots, P_{\text {corN }}^{\mathrm{INP}}$, determined with a delay of $T_{\mathrm{s}}$ due to the $\mathrm{SH}$. The correction power of the $\mathrm{CA} i$ characterizes the maximum interchange power for the CA $i$ among the cooperating CAs with a different sign of $A C E_{i}$, and is included as

$$
A C E_{i}^{\mathrm{INP}}=\left(B_{i} \Delta f_{i}+\Delta P_{i}\right)-P_{\mathrm{cor} i}^{\mathrm{INP}},
$$

where the terms in brackets denote $A C E_{i}$.

Moreover, only CAs with different signs of demand power, i.e., $\operatorname{sign}\left(P_{\mathrm{d} i}\right) \neq \operatorname{sign}\left(P_{\mathrm{d} j}\right)$, can net the demand for balancing energy. If two or more cooperating CAs are "short", then CBRR is used instead of the INP and vice versa [23]. Therefore, the cooperating CAs must be "short" and "long" in order to net the demand power through the INP. Hence, the balancing energy in CAs that net the balancing energy from the cooperating CAs can be reduced, and simultaneously the RR is released. The $P_{\text {cor } i}^{\mathrm{INP}}, P_{\mathrm{cor} j}^{\mathrm{INP}}, \ldots, P_{\mathrm{corN}}^{\mathrm{INP}}$ is determined by the INP optimization module, considering numerous target functions, as given in [20].

Considering N CAs, then the $P_{\text {cori }}^{\mathrm{INP}}$ is, for the CA $i$, expressed as

$$
P_{\mathrm{cor} i}^{\mathrm{INP}}=-P_{\mathrm{d} i} K_{i}^{\mathrm{INP}}+P_{\mathrm{d} j} K_{j}^{\mathrm{INP}}+, \ldots,+P_{\mathrm{d} N} K_{N}^{\mathrm{INP}} .
$$

Considering (4), then the $P_{\text {cor } i}^{\mathrm{INP}}$ between N CAs is, for the CA $i$, expressed as

$$
P_{\text {cor } i}^{\mathrm{INP}}=-\left(\Delta P_{\mathrm{L} i}-B_{i} \Delta f_{i}\right) K_{i}^{\mathrm{INP}}+\left(\Delta P_{\mathrm{L} j}-B_{j} \Delta f_{j}\right) K_{j}^{\mathrm{INP}}+, \ldots,+\left(\Delta P_{\mathrm{LN}}-B_{N} \Delta f_{N}\right) K_{N}^{\mathrm{INP}} .
$$

In this way, the correction power of the CA $i$ compensates the load variation that is varied by the frequency variation of the cooperating CAs. From a system point of view, this corresponds to additional frequency-based feedback and cross-couplings with cooperating CAs, which inseparably changes the eigendynamics of the CA $i$ [21].

\section{3. $C B R R$}

The basic principle of the CBRR operation and a steady-state correction-value calculation with the CBRR for three CAs is given in [22]. The same control-demand concept is used for the CBRR as is currently used for the INP, and a schematic block diagram representation is shown in Figure 4 [3]. Similar to INP, N CAs can be connected via the virtual tie-lines, i.e., they can all activate the CBRR via the activation factors $K_{i}^{\mathrm{CBRR}}, K_{j}^{\mathrm{CBRR}}$, $\ldots, K_{N}^{\mathrm{CBRR}}$ marked with the green rectangle. Note that the factor $K_{j}^{\mathrm{CBRR}}$ represents the size of the CBRR activation for the $C A j$ in the $C A i$, where $K_{j}^{C B R R}=0$ determines that the possible CBRR activation is equal to $0 \%$, whereas $K_{j}^{\mathrm{CBRR}}=1$ determines that the possible CBRR activation is equal to $100 \%$. Moreover, the values of $K_{i}^{\mathrm{CBRR}}, K_{j}^{\mathrm{CBRR}}, \ldots, K_{N}^{\mathrm{CBRR}}$ can 
be different. The cooperating CAs are connected to the "red" summator, forming virtual tie-lines, as shown in Figure 4.

The input variables to the "red" summator are the demand powers of the cooperating CAs, i.e., $P_{\mathrm{d} i}, P_{\mathrm{d} j}, \ldots, P_{\mathrm{d} N}$. The demand power of the CA $i$ characterizes the maximum activation power for the CA $i$ among the cooperating CAs, defined as (3), according to $[3,29]$.

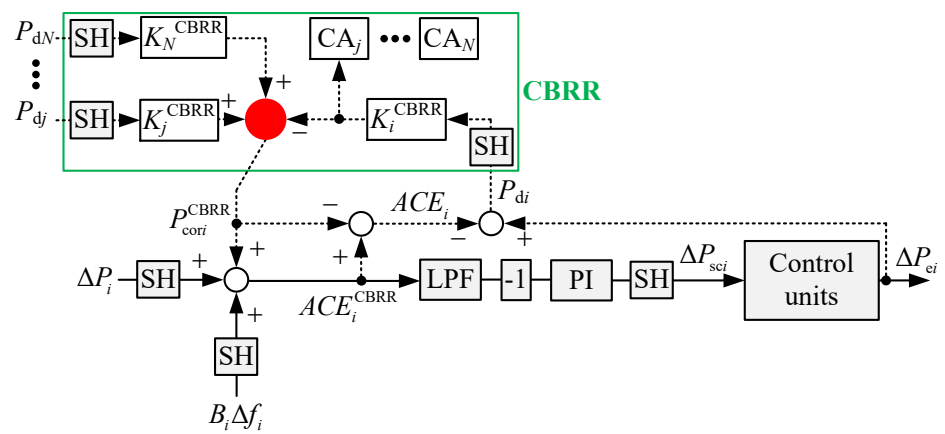

Figure 4. Schematic block diagram representation of the LFC in the CA $i$ (solid line) with the CBRR (solid and dotted line).

Similar to INP, by introducing (1) and (2) in (3), (4) is obtained.

The power imbalance between generation and load in addition to $K_{i}^{\mathrm{CBRR}} P_{\mathrm{d} i}$ from the $\mathrm{CA} i, K_{j}^{\mathrm{CBRR}} P_{\mathrm{d} j}$ from the CA $j, \ldots, K_{N}^{\mathrm{CBRR}} P_{\mathrm{d} N}$ from the CA $N$ is, for the CA $i$, defined as

$$
A C E_{i}^{\mathrm{CBRR}}=B_{i} \Delta f_{i}+\Delta P_{i}-K_{i}^{\mathrm{CBRR}} P_{\mathrm{d} i}+\left(K_{j}^{\mathrm{CBRR}} P_{\mathrm{d} j}+, \ldots,+K_{N}^{\mathrm{CBRR}} P_{\mathrm{d} N}\right) .
$$

The output variables of the "red" summator are the correction powers of the cooperating CAs, i.e., $P_{\text {cori }}^{\mathrm{CBRR}}, P_{\mathrm{cor} j}^{\mathrm{CBRR}}, \ldots, P_{\mathrm{corN}}^{\mathrm{CBRR}}$, determined with a delay of $T_{\mathrm{S}}$ due to the $\mathrm{SH}$. The correction power of the CA $i$ characterizes the maximum activation power for the $\mathrm{CA} i$ among the cooperating CAs with matching sign of $A C E_{i}$, and is included as

$$
A C E_{i}^{\mathrm{CBRR}}=\left(B_{i} \Delta f_{i}+\Delta P_{i}\right)+P_{\mathrm{cor} i}^{\mathrm{CBRR}},
$$

where the terms in brackets denote $A C E_{i}$.

Moreover, only CAs with matching sign of demand power, i.e., $\operatorname{sign}\left(P_{\mathrm{d} i}\right)=\operatorname{sign}\left(P_{\mathrm{d} j}\right)$, can activate the demand for balancing energy. If any of the cooperating CAs are "long" and the others are "short", then INP is used instead of the CBRR and vice versa [21]. Therefore, the cooperating CAs must be either "short" or "long", depending on whether a positive or negative CBRR is activated. Hence, the balancing energy in CAs that activates the balancing energy in the cooperating CAs can be reduced, and simultaneously the RR is released. The $P_{\mathrm{cor} i}^{\mathrm{CBRR}}, P_{\mathrm{cor} j}^{\mathrm{CBRR}}, \ldots, P_{\mathrm{corN}}^{\mathrm{CBRR}}$ is determined by the CBRR optimization module, considering numerous target functions, as given in [22].

Considering N CAs, then the $P_{\mathrm{cor} i}^{\mathrm{CBRR}}$ is, for the CA $i$, expressed as

$$
P_{\text {cor } i}^{\mathrm{CBRR}}=-P_{\mathrm{d} i} K_{i}^{\mathrm{CBRR}}+P_{\mathrm{d} j} K_{j}^{\mathrm{CBRR}}+, \ldots,+P_{\mathrm{d} N} K_{N}^{\mathrm{CBRR}} .
$$

Considering (4), then the $P_{\text {cori } i}^{\mathrm{CBRR}}$ between $N$ CAs is, for the CA $i$, expressed as

$$
P_{\text {cori }}^{\mathrm{CBRR}}=-\left(\Delta P_{\mathrm{L} i}-B_{i} \Delta f_{i}\right) K_{i}^{\mathrm{CBRR}}+\left(\Delta P_{\mathrm{L} j}-B_{j} \Delta f_{j}\right) K_{j}^{\mathrm{CBRR}}+, \ldots,+\left(\Delta P_{\mathrm{L} N}-B_{N} \Delta f_{N}\right) K_{N}^{\mathrm{CBRR}} .
$$

Similar to the INP, the correction power of the CA $i$ compensates the load variation that is varied by the frequency variation of the cooperating CAs. From a system point of view, this corresponds to an additional frequency-based feedback and cross-couplings with cooperating CAs, which inseparably changes the eigendynamics of the CA $i$ [23]. 


\subsection{Simultaneous Operation of the INP and the CBRR}

In the cooperating CAs, $\Delta P_{\mathrm{L} i}$ changes randomly and continuously. Consequently, cases of $\Delta P_{\mathrm{d} i}$ sign changes can occur, resulting in a continuous switching between the INP and the CBRR, which causes undesirable $\Delta P_{\text {cori }}$ sign changes. Such a situation might occur when the signs of $\Delta P_{\mathrm{d} i}$ and $\Delta P_{\mathrm{d} j}$ are changed with a short time delay. Therefore, a function for $P_{\text {cori }}$ adjustment is proposed as one of the contributions of this article.

A schematic block diagram representation for $P_{\text {cori }}$ adjustment in relation to $P_{\mathrm{d} i}$ of the CA $i$ is shown in Figure 5. The signs of the two successive samples, i.e., $P_{\mathrm{d} i, k-1}$ and $P_{\mathrm{d} i, k}$, are compared with the relational operator, whose output is connected to a switch, marked with " $c$ ". Two states are possible, i.e.,

- State 1: $\operatorname{sign}\left(P_{\mathrm{d} i, k-1}\right)=\operatorname{sign}\left(P_{\mathrm{d} i, k}\right)$ and

- $\quad$ State 2: $\operatorname{sign}\left(P_{\mathrm{d} i, k-1}\right) \neq \operatorname{sign}\left(P_{\mathrm{d} i, k}\right)$.

For State 1 , the switch position is " 1 " and the output variable is $P_{\text {cori }}^{\mathrm{ADJ}}=P_{\text {cori }}$. For State 2, the switch position is " 2 " and the output variable is $P_{\text {cori }}^{\mathrm{ADJ}}=0$.

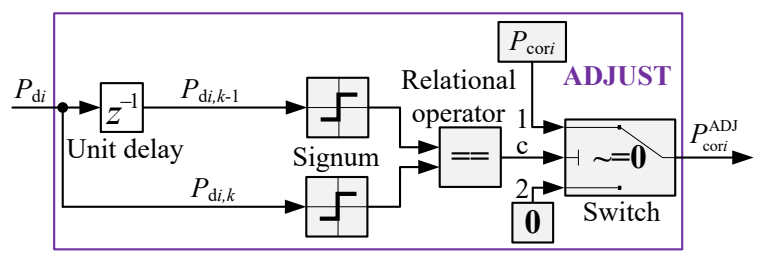

Figure 5. Schematic block diagram representation for $P_{\text {cori }}$ adjustment in the CA $i$.

The implementation of the $P_{\text {cori }}$ adjustment in the INP and the CBRR framework is shown in Figure 6. The simultaneous operation of the INP and the CBRR, considering the $P_{\text {cori }}$ adjustment, can be described using the pseudo-code, as shown in Alogorithm 1.
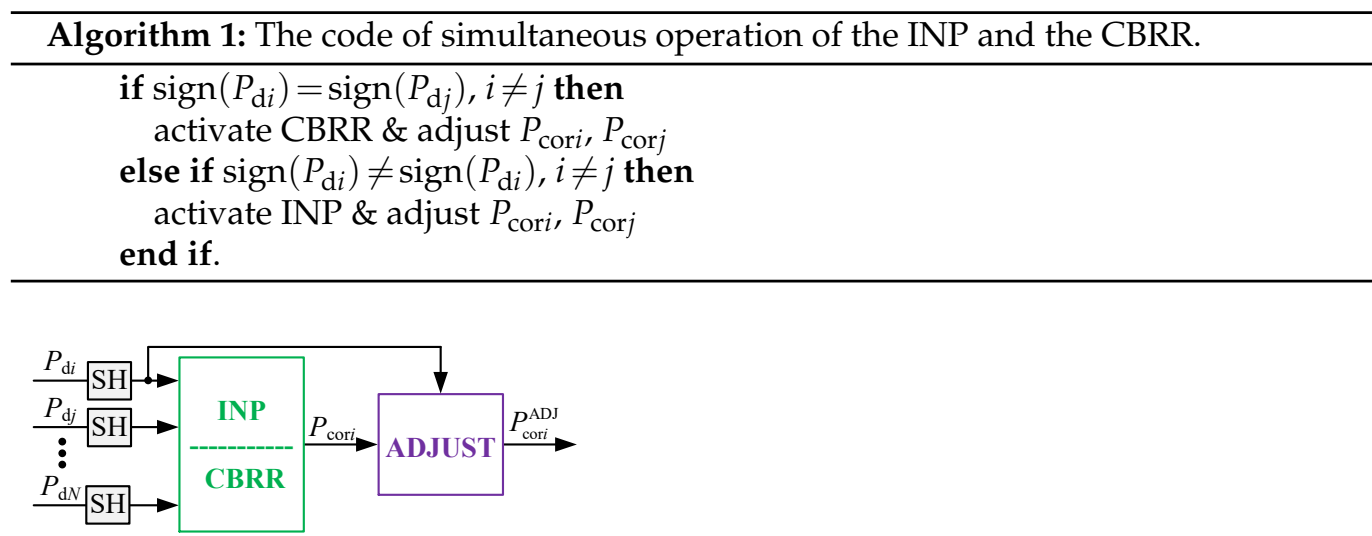

Figure 6. Schematic block diagram representation for $P_{\text {cori }}$ adjustment with the INP and the CBRR in the CA $i$.

An example of the simultaneous operation of the INP and the CBRR, with and without the $P_{\text {cori }}$ adjustment, is shown in Figure 7. The values of the loads were set in such a way that the simultaneous operation of the INP and the CBRR was possible. In the time interval of 80-100 s, the INP operated between $\mathrm{CA}_{1}-\mathrm{CA}_{3}$ and $\mathrm{CA}_{2}-\mathrm{CA}_{3}$, whereas the CBRR operated between $\mathrm{CA}_{1}-\mathrm{CA}_{2}$. During this time interval, $P_{\text {cori }}$ due to the INP was possible in all three CAs, whereas $P_{\text {cori }}$ due to CBRR was possible only in $\mathrm{CA}_{1}$ and $\mathrm{CA}_{2}$. At $t=100 \mathrm{~s}$, a simultaneous step change of the load in $\mathrm{CA}_{1}$ and $\mathrm{CA}_{3}$ was applied, whereas, in $\mathrm{CA}_{2}$, it was applied with a delay, i.e., at $t=100.05 \mathrm{~s}$, as seen at $t=102 \mathrm{~s}$ due to SH with $T_{\mathrm{s}}=2 \mathrm{~s}$. Time responses of $P_{\mathrm{d} i}$ and $P_{\text {cori }}$ at $t=100 \mathrm{~s}$ with one step-size activation of the INP in $C_{1}$ and $\mathrm{CA}_{3}$, and the CBRR in $\mathrm{CA}_{3}$ is clearly seen in Figure $7 \mathrm{a}$ (without $P_{\text {cori }}$ adjustment). Note that $P_{\text {cori }}$ due to CBRR should be zero in $\mathrm{CA}_{3}$. Additionally, at $t=102 \mathrm{~s}$, one step-size 
activation of the CBRR in $\mathrm{CA}_{2}$ is also seen. However, in Figure $7 \mathrm{~b}$ (with $P_{\text {cori }}$ adjustment), at $t=100 \mathrm{~s}$ and $t=102 \mathrm{~s}$, the value of $P_{\text {cor } i}$ was zero in all CAs. In this way, delayed $P_{\mathrm{d} i}$ sign changes have no impact on the switching between the INP and the CBRR. Moreover, the $P_{\text {cori }}$ variation is significantly reduced using the proposed adjustment.
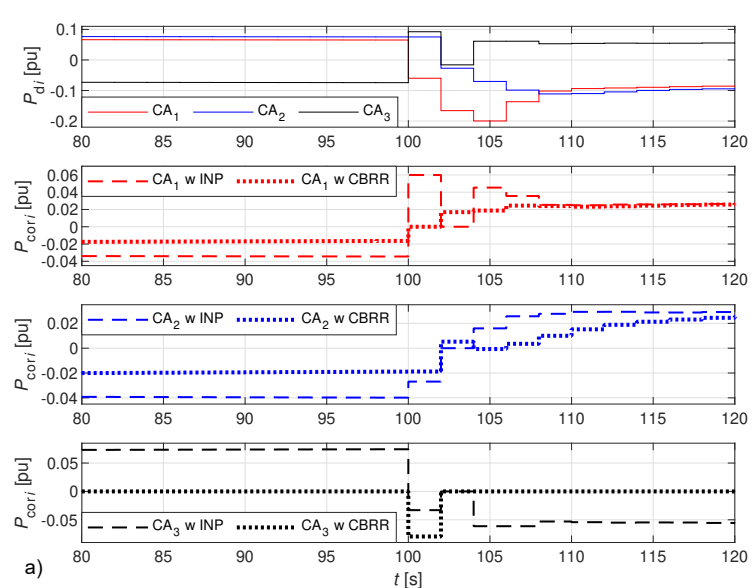
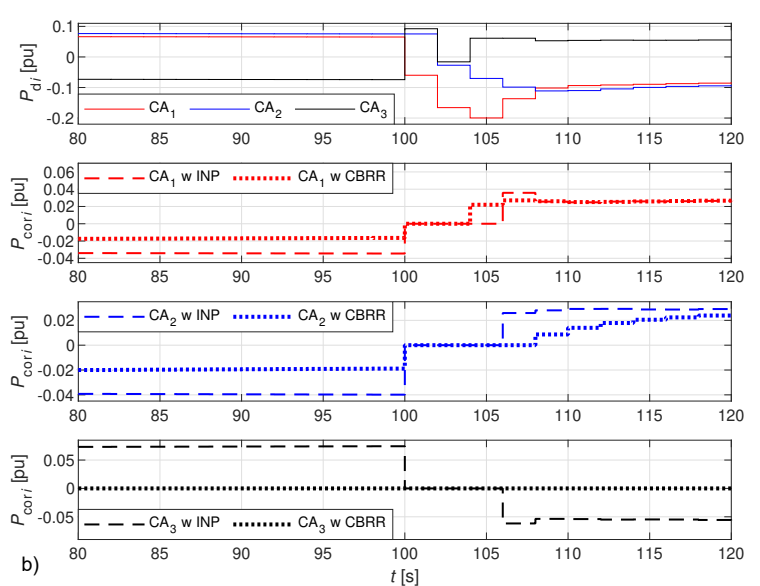

Figure 7. Time responses of $P_{\mathrm{d} i}$ and $P_{\text {cor } i}$ for a three-CA testing system without $P_{\text {cor } i}$ adjustment (a) and with $P_{\text {cor } i}$ adjustment (b), where "w INP" is with the INP and "w CBRR" is with the CBRR.

\section{Indicators for Evaluation of LFC, INP, and CBRR Provision}

The impact of the simultaneous operation of the INP and CBRR on the frequency quality, the LFC, and performance is evaluated using 15-min averages [1].

\subsection{Performance Indicators}

Frequency quality is evaluated with the standard deviation of $\Delta f_{i}$, denoted as $\sigma_{\Delta f i}$. In addition, the LFC and performance is evaluated with the standard deviation of $A C E_{i}$, denoted as $\sigma_{A C E i}[1,30]$.

\section{2. $\mathrm{RoCoF}$}

RoCoF is the time derivative of the power system's frequency, i.e., $\frac{d f_{i}}{d t}[31]$. The mean value of $\mathrm{RoCoF}_{i}$, denoted as $\mu_{\mathrm{RoCoF}_{i}}$, is evaluated individually for positive and negative values, denoted as $\mu_{\mathrm{RoCoF}_{i+}}$ and $\mu_{\mathrm{RoCoF}_{i-}}$.

\subsection{Standard Deviation and Mean Value of RRs}

RRs assist in active power balance to correct the imbalance in the transmission grid and lead the power system frequency to the normal frequency range [27]. The standard deviation and mean value of $\Delta P_{\mathrm{sc} i}$ are calculated individually for positive and negative values, denoted as $\sigma_{\Delta P_{\mathrm{sci}}+}, \sigma_{\Delta P_{\mathrm{sci}-}}$ and $\mu_{\Delta P_{\mathrm{sci} i}}, \mu_{\Delta P_{\mathrm{sci}-}}$.

\subsection{Balancing Energy}

The balancing energy enables TSOs to cost-effectively compensate for power and voltage variation in the transmission grid [2]. It is the actual electrical control power that is, for a particular period of time, calculated as $\Delta W_{\mathrm{e} i}=\int_{0}^{t} \Delta P_{\mathrm{e} i} d t$. Individual positive and negative values, denoted as $\Delta W_{\mathrm{e} i+}$ and $\Delta W_{\mathrm{e} i-}$, are calculated.

\subsection{Unintended Exchange of Energy}

The unintended exchange of energy is determined by the difference between interchange power variation and correction power [2] that is, for a particular period of time, calculated as $\Delta W_{\mathrm{un} i}=\int_{0}^{t}\left(\Delta P_{i}-P_{\text {cori }}\right) d t$. Individual positive and negative values, denoted as $\Delta W_{\mathrm{uni+}}$ and $\Delta W_{\mathrm{uni-}}$, are calculated. 


\subsection{Energy Exchange}

The energy exchange through the INP and CBRR is defined as the actual interchanged or activated power between cooperating CAs [32], that is, for a particular period of time, calculated as $W_{\text {cor } i}=\int_{0}^{t} P_{\text {cori }} d t$. Additionally, positive and negative values are calculated, individually for the INP, denoted as $W_{\text {cori+ }}^{\mathrm{INP}}, W_{\text {cori- }}^{\mathrm{INP}}$, and individually for the CBRR, denoted as $W_{\text {cori } i+}^{\mathrm{CBRR}}, W_{\text {cori- }}^{\mathrm{CBRR}}$.

\section{Dynamic Simulations}

A three-CA test system was used for the dynamic simulations, where $C A_{1}-C A_{2}$ and $\mathrm{CA}_{2}-\mathrm{CA}_{3}$ were connected by physical tie-lines, whereas $\mathrm{CA}_{1}-\mathrm{CA}_{3}$ were not connected with a tie-line. Moreover, all three $\mathrm{CAs}$ were connected with virtual tie-lines due to the INP and the CBRR. A Matlab/SIMULINK model was used, where the dynamic simulations were performed with a $50 \mathrm{~ms}$ step size.

\subsection{Dynamic Model}

\subsubsection{Structure}

The basic schematic block diagram representation of a single CA, characterized with a linearized, low-order, time-invariant model, is shown in Figures 8 and $9[29,33]$. Note that the INP and CBRR implementation is not shown. The generator-load dynamic is described by the rotor inertia $H_{i}$ and the damping $D_{i}$. Moreover, three different types of the turbine-governor systems were considered, i.e., hydraulic, steam reheat, and steam non-reheat. A constant droop characteristic $R_{\mathrm{n} i}$ was assumed. In addition, the ramping rate and the participation factors $\alpha_{\mathrm{n} i}$ of the control units were also taken into account. The tie-line between the connected CAs is described by the synchronizing coefficient $T_{i j}$ [34]. Furthermore, a 1st-order LPF is modeled by a time constant $T_{\mathrm{LPF} i}$, while the PI controller is modeled by a gain $K_{\mathrm{r} i}$ and a time constant $T_{\mathrm{r} i}$.

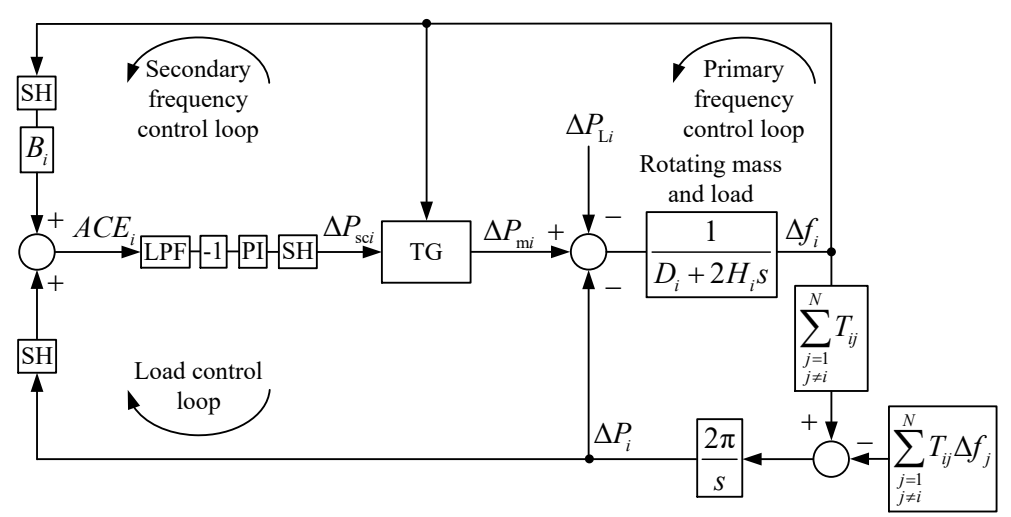

Figure 8. Schematic block diagram representation of the CA $i$ without the INP and the CBRR.

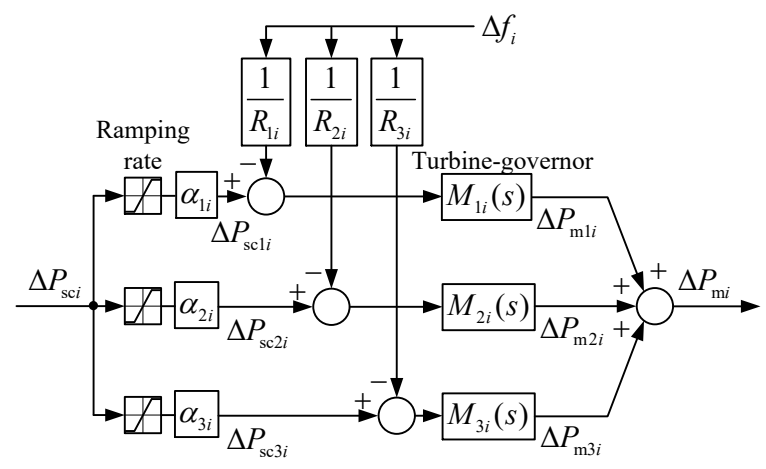

Figure 9. Schematic block diagram representation of the turbine-governor system with primary frequency control of the CA $i$. 


\subsubsection{Parameters}

Common parameter values were set for a three-CA test system as given in Table $1[28,29]$. Note that the frequency-bias coefficient was determined as a constant, i.e., $B_{i}=1 / R_{1 i}+$ $1 / R_{2 i}+1 / R_{3 i}+D_{i}$. The model parameters were set equally for all three CAs. One cycle of the LFC, INP, and CBRR was incorporated with $T_{\mathrm{s}}=2 \mathrm{~s}$.

Table 1. Parameter values for a three-CA test system.

\begin{tabular}{cccc}
\hline Parameter & Value & Parameter & Value \\
\hline$H_{i}$ & $0.1 \mathrm{pu} \mathrm{s}$ & $\alpha_{\mathrm{n} i}$ & $1 / 3$ \\
$D_{i}$ & $0.01 \mathrm{pu} / \mathrm{Hz}$ & $K_{\mathrm{r} i}$ & 0.3 \\
$T_{i j}$ & $1 / 30 \mathrm{pu} / \mathrm{Hz}$ & $R_{\mathrm{n} i}$ & $3 \mathrm{~Hz} / \mathrm{pu}$ \\
$T_{\mathrm{LPF} i}$ & $0.3 \mathrm{~s}$ & $T_{\mathrm{r} i}$ & $30 \mathrm{~s}$ \\
\hline ramp rate & value & ramp rate & value \\
\hline hydraulic & $\pm 100 \mathrm{puMW} / \mathrm{min}$ & rehat & $\pm 10 \mathrm{puMW} / \mathrm{min}$ \\
non-reheat & $\pm 20 \mathrm{puMW} / \mathrm{min}$ & - & - \\
\hline
\end{tabular}

\subsection{Test Cases}

The maximum possible compensation with the INP and CBRR was considered. Dynamic simulations were performed so that the loads of individual CAs were altered during the simulation. In addition, two types of test cases were performed, i.e., step change of the load and the random load variation.

Moreover, the inertia time constant $H_{i}$, the tie-line parameter $T_{i j}$, and the droop characteristic $R_{i}$ have a considerable impact on frequency quality according to $[35,36]$. Therefore, different values of $H_{i}, T_{i j}$, and $R_{i}$ were used to show the impact of the simultaneous operation of the INP and CBRR on the indicators for evaluation of LFC, INP, and CBRR provision.

\subsubsection{Step Change of Load}

The values of the loads were set in such a way that the simultaneous operation of the INP and the CBRR was possible. At $t=0 \mathrm{~s}$, a simultaneous step change of the loads was applied and the magnitudes were set as $\Delta P_{\mathrm{L} 1}=0.06 \mathrm{pu}, \Delta P_{\mathrm{L} 2}=0.07 \mathrm{pu}$ and $\Delta P_{\mathrm{L} 3}=-0.08 \mathrm{pu}$. In addition, at $t=100 \mathrm{~s}$, the magnitudes were set as $\Delta P_{\mathrm{L} 1}=-0.06 \mathrm{pu}$ and $\Delta P_{\mathrm{L} 3}=0.08 \mathrm{pu}$, whereas, at $t=100.05 \mathrm{~s}$, the magnitude was set as $\Delta P_{\mathrm{L} 2}=-0.07 \mathrm{pu}$. The resulting load is shown in Figure 10a. Consequently, the INP operated between $\mathrm{CA}_{1}-\mathrm{CA}_{3}$ and $\mathrm{CA}_{2}-\mathrm{CA}_{3}$, while the $\mathrm{CBRR}$ operated only between $\mathrm{CA}_{1}-\mathrm{CA}_{2}$. Note that this case is used in Section 2.4 .

a)

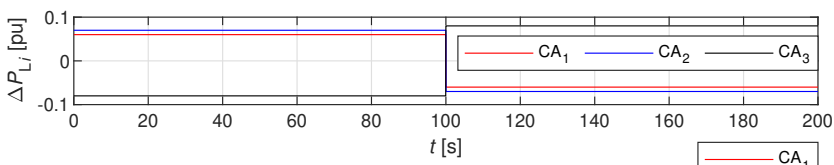

b1)

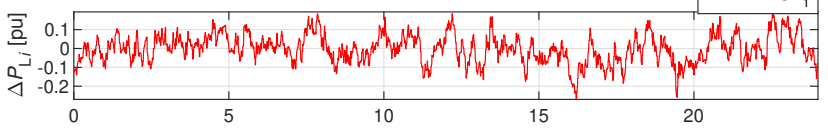

b2)

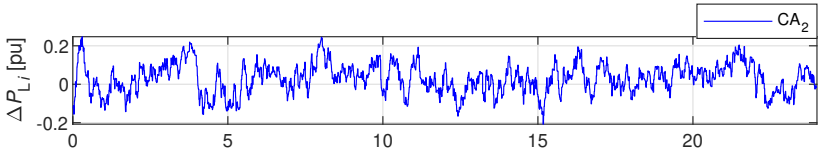

b3)

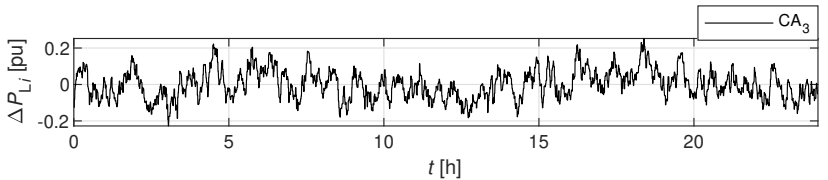

Figure 10. Step changes of $\Delta P_{\mathrm{L} i}(\mathbf{a})$ and random $\Delta P_{\mathrm{L} i}$ variations (b1-b3) for a three CA test system. 


\subsubsection{Random Load Variation}

The random load was modeled as a linear, stochastic, time-invariant, first-order system with two components [37]. A low-frequency component captures the trend changes with a quasi-period of 10-30 min, whereas the residual component captures fluctuations with a quasi-period of several minutes. Measurements of an open-loop ACE in an undisclosed CA were used to determine the model parameters. The resulting normalized load for a three-CA test system with different signs is shown in Figure 10(b1-b3), where the random load was changed every $60 \mathrm{~s}$ for $24 \mathrm{~h}$. The statistical parameters of the random loads for all three CAs are given in Table 2, where $\mu_{\mathrm{L} i}$ and $\sigma_{\mathrm{L} i}$ denote the meanvalue and the standard deviation of the $i$-th load, while the correlation between the $i$-th and $j$-th loads is denoted as $\rho_{\mathrm{L} i j}$. The correlation $\rho_{\mathrm{L} i j}$ is extremely small, which allows both the INP and CBRR to operate simultaneously.

Table 2. Statistical parameters of random loads.

\begin{tabular}{ccc}
\hline$\mu_{\mathbf{L} 1}\left[\mathbf{p u} \cdot \mathbf{1 0}^{3}\right]$ & $\boldsymbol{\mu}_{\mathrm{L} 2}\left[\mathbf{p u} \cdot \mathbf{1 0}^{3}\right]$ & $\mu_{\mathrm{L} 3}\left[\mathbf{p u} \cdot \mathbf{1 0 ^ { 3 }}\right]$ \\
\hline-2.501 & 29.526 & -0.693 \\
\hline$\sigma_{\mathrm{L} 1}\left[\mathrm{pu} \cdot 10^{3}\right]$ & $\sigma_{\mathrm{L} 2}\left[\mathrm{pu} \cdot 10^{3}\right]$ & $\sigma_{\mathrm{L} 3}\left[\mathrm{pu} \cdot 10^{3}\right]$ \\
\hline 74.891 & 76.890 & 78.827 \\
\hline$\rho_{\mathrm{L} 12}$ & $\rho_{\mathrm{L} 23}$ & $\rho_{\mathrm{L} 31}$ \\
\hline-0.056 & 0.059 & 0.004 \\
\hline
\end{tabular}

\section{Results}

Dynamic simulations with and without the INP and CBRR were performed for a three-CA test system. The impact of the simultaneous operation of the INP and CBRR on the frequency quality, the LFC, and performance was evaluated with the obtained results. Note that the results shown in this section refer to a three-CA test system, whereas the basic principle is applicable to N CAs as shown in Section 2. In addition, the results cannot be generalized to the dynamics of the INP and CBRR.

\subsection{Step Change of Load}

The time responses to the step change of $\Delta P_{\mathrm{L} i}$ are shown in Figures 11-13. In Figure 11 (left), it is clear that the frequency deviations $\Delta f_{i}$ in all three CAs appeared following a step change of $\Delta P_{\mathrm{L} i}$ that was applied. After the first step change, $\Delta f_{1}$ and $\Delta f_{2}$ were negative due to the positive step change of $\Delta P_{\mathrm{L} i}$, whereas $\Delta f_{3}$ was positive due to the negative step change of $\Delta P_{\mathrm{L} i}$. Note that after the second step change, the signs were opposite. The primary frequency control decreased $\left|\Delta f_{i}\right|$ in about $15-25 \mathrm{~s}$ after the step change of $\Delta P_{\mathrm{L} i} ;$ then, LFC decreased $\left|\Delta f_{i}\right|$ slowly. The results show that the impact of the INP and CBRR on $\Delta f_{i}$ is not significant.

The impact of the INP and CBRR is shown more obviously in Figures 11 (right) and 12 (left). In all three CAs, the values of $A C E_{i}, \Delta P_{\mathrm{sc} i}$ and $\Delta P_{\mathrm{e} i}$ were decreased with the INP and CBRR. Furthermore, the INP and CBRR clearly increased $\Delta P_{i}$, due to the increased tie-line power flow between the CAs.

The signs of $P_{\mathrm{d} i}$ and $P_{\text {cori }}$ are opposite, as shown in Figure 12 (right). A $2 \mathrm{~s}$ time delay is seen, due to SH with $T_{\mathrm{s}}=2 \mathrm{~s}$. Note that the time responses of $P_{\mathrm{d} i}$ and $P_{\text {cori }}$ were already described in Section 2.4 and Figure $7 \mathrm{~b}$, where the same case was performed.

The time responses $A C E_{i}$ and $\triangle P_{\mathrm{sc} i}$ with and without the $P_{\text {cori }}$ adjustment function are shown in Figure 13. Without the $P_{\text {cori }}$ adjustment, $A C E_{i}$ was increased and $\Delta P_{\text {sci }}$ was undesirably increased, due to switching between the INP and the CBRR, which caused undesirable $\Delta P_{\text {cori }}$ sign changes. Clearly, in all three CAs, the values of $A C E_{i}$ and $\Delta P_{\text {sci }}$ were decreased with the $P_{\text {cori }}$ adjustment. Note that, in Figure 13, the same example is performed as described in Section 4.2.1 and shown in Figures 11 and 12. The difference can 
be seen because, in Figures 11 and 12, the comparison without and with the INP and CBRR is shown, whereas, in Figure 13, the comparison without and with the $P_{\text {cori }}$ adjustment is shown.
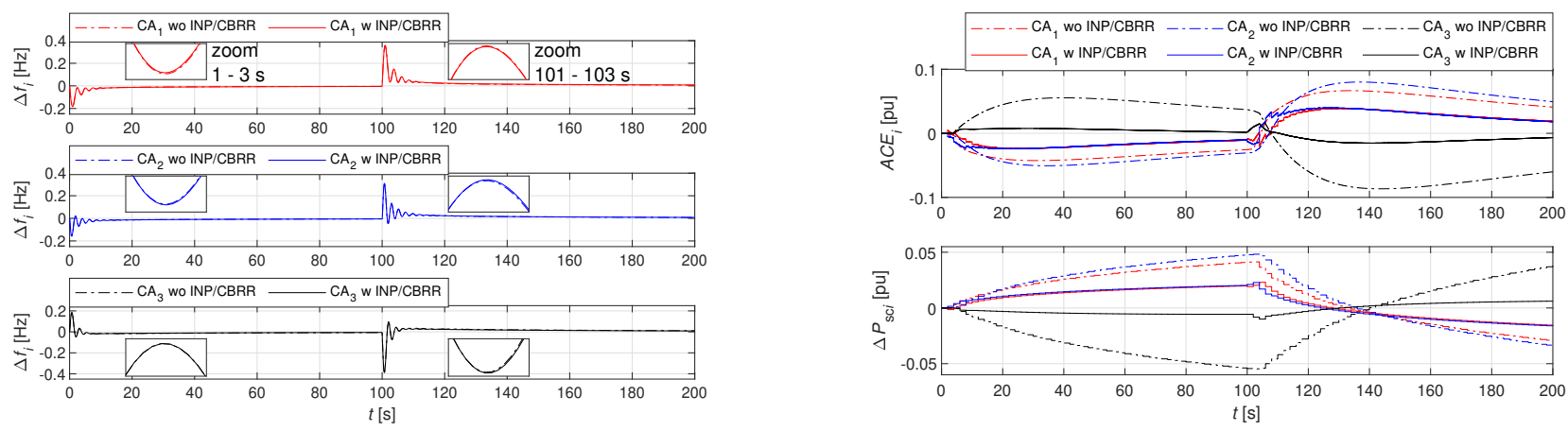

Figure 11. Time responses of $\Delta f_{i}$ (left) and time responses of $A C E_{i}$ and $\Delta P_{\mathrm{sc} i}$ (right) for a three-CA test system, where "wo INP/CBRR" is without and "w INP/CBRR" is with the INP and CBRR.
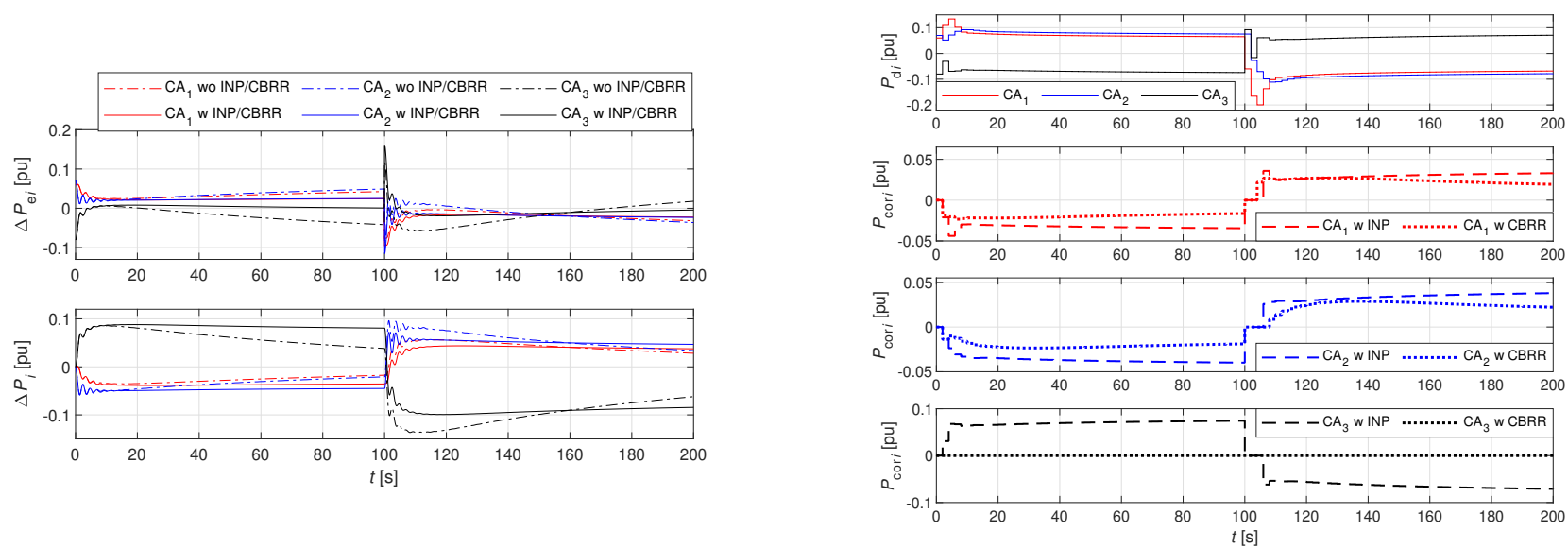

Figure 12. Time responses of $\Delta P_{\mathrm{e} i}$ and $\Delta P_{i}$ (left) and time responses of $P_{\mathrm{d} i}$ and $P_{\text {cori }}$ (right) for a three-CA test system, where "wo INP/CBRR" is without and "w INP/CBRR" is with the INP and CBRR, whereas "w INP" is with the INP and "w CBRR" is with the CBRR.
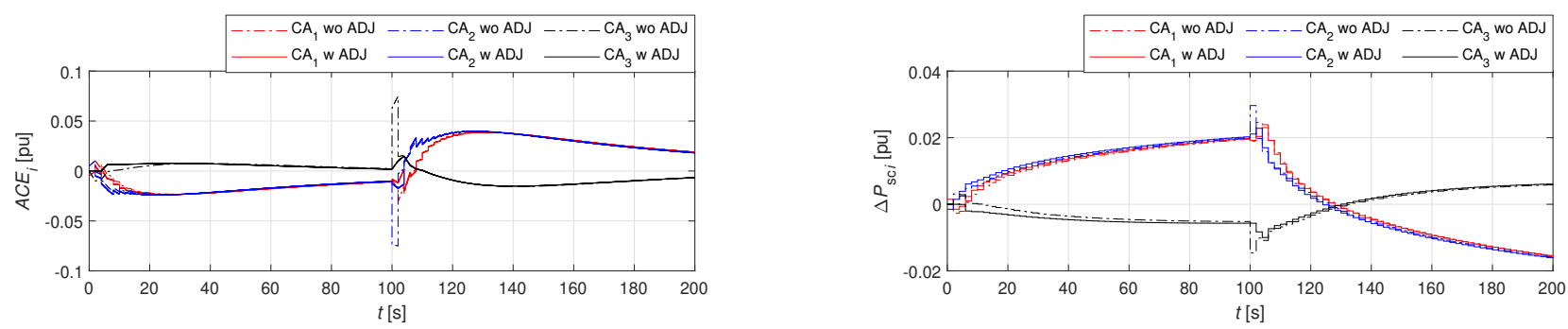

Figure 13. Time responses of $A C E_{i}$ (left) and time responses of $\triangle P_{\mathrm{sc} i}$ (right) for a three-CA test system, where "wo ADJ" is without and "w ADJ" is with the $P_{\text {cori }}$ adjustment function.

\subsection{Random Load Variation}

Simulations of the simultaneous operation of the INP and CBRR were performed to show the impact of an individual mechanism. The simulations were also performed separately, i.e., operation of only the INP or only the CBRR. The results are given in Tables 3-5.

There are no unambiguous conclusions about the impact of the mechanisms on $\sigma_{\Delta f i}$, and the differences are extremely small, as shown in Table 3. However, both mechanisms reduce $\sigma_{A C E i}$, INP slightly more than CBRR. The reduction is most pronounced when both mechanisms operate simultaneously. 
Table 3. Performance indicators, RoCoF, mean value, and standard deviation of RRs.

\begin{tabular}{|c|c|c|c|c|c|c|c|c|}
\hline \multirow{2}{*}{ Indicator } & \multicolumn{4}{|c|}{$\sigma_{\Delta f i}[\mathrm{mHz}]$} & \multicolumn{4}{|c|}{$\sigma_{A C E i}\left[\mathrm{pu} \cdot 10^{3}\right]$} \\
\hline & wo & INP/CBRR & INP & CBRR & wo & INP/CBRR & INP & CBRR \\
\hline $\mathrm{CA}_{1}$ & 5.263 & 5.256 & 5.251 & 5.272 & 8.873 & 6.310 & 6.654 & 8.649 \\
\hline $\mathrm{CA}_{2}$ & 5.252 & 5.256 & 5.250 & 5.260 & 9.612 & 5.844 & 7.033 & 8.185 \\
\hline $\mathrm{CA}_{3}$ & 5.265 & 5.267 & 5.273 & 5.260 & 8.823 & 6.063 & 6.439 & 8.281 \\
\hline \multirow{2}{*}{ indicator } & \multicolumn{4}{|c|}{$\mu_{\mathrm{RoCoFi} i+}[\mathrm{mHz} / \mathrm{s}]$} & \multicolumn{4}{|c|}{$\mu_{\mathrm{RoCoF} i-}[\mathrm{mHz} / \mathrm{s}]$} \\
\hline & wo & INP/CBRR & INP & CBRR & wo & INP/CBRR & INP & CBRR \\
\hline $\mathrm{CA}_{1}$ & 0.682 & 0.606 & 0.611 & 0.677 & -0.724 & -0.645 & -0.674 & -0.692 \\
\hline $\mathrm{CA}_{2}$ & 0.879 & 0.776 & 0.832 & 0.818 & -1.233 & -1.039 & -1.072 & -1.193 \\
\hline $\mathrm{CA}_{3}$ & 0.752 & 0.690 & 0.722 & 0.711 & -0.841 & -0.740 & -0.743 & -0.830 \\
\hline \multirow{2}{*}{ indicator } & \multicolumn{4}{|c|}{$\mu_{P \mathrm{sc} i+}\left[\mathrm{pu} \cdot 10^{3}\right]$} & \multicolumn{4}{|c|}{$\mu_{P \mathrm{sc} i-}\left[\mathrm{pu} \cdot 10^{3}\right]$} \\
\hline & wo & INP/CBRR & INP & CBRR & wo & INP/CBRR & INP & CBRR \\
\hline $\mathrm{CA}_{1}$ & 46.17 & 36.60 & 31.20 & 46.38 & -47.08 & -28.87 & -29.69 & -45.97 \\
\hline $\mathrm{CA}_{2}$ & 58.62 & 32.95 & 43.77 & 49.68 & -41.10 & -23.06 & -22.40 & -31.63 \\
\hline $\mathrm{CA}_{3}$ & 53.09 & 35.58 & 39.46 & 47.99 & -50.11 & -29.81 & -31.01 & -44.21 \\
\hline \multirow{2}{*}{ indicator } & \multicolumn{4}{|c|}{$\sigma_{P \mathrm{sci}}\left[\mathrm{pu} \cdot 10^{3}\right]$} & \multicolumn{4}{|c|}{$\sigma_{P \mathrm{sc} i-}\left[\mathrm{pu} \cdot 10^{3}\right]$} \\
\hline & wo & INP / CBRR & INP & CBRR & wo & INP/CBRR & INP & CBRR \\
\hline $\mathrm{CA}_{1}$ & 36.68 & 26.35 & 28.83 & 32.17 & 40.43 & 18.55 & 23.49 & 34.44 \\
\hline $\mathrm{CA}_{2}$ & 45.13 & 25.70 & 39.43 & 31.00 & 29.59 & 18.25 & 25.95 & 24.32 \\
\hline $\mathrm{CA}_{3}$ & 39.36 & 26.98 & 32.78 & 31.03 & 31.67 & 20.14 & 24.18 & 26.12 \\
\hline
\end{tabular}

Legend: wo-without the INP/CBRR, INP/CBRR—simultaneous operation, INP—separate operation, CBRRseparate operation.

Both mechanisms reduce the $\left|\mu_{\text {RoCoFi+ }}\right|$ and $\left|\mu_{\text {RoCoFi- }}\right|$, with the reduction being most pronounced when both mechanisms operate simultaneously according to Table 3 . However, there are no unambiguous conclusions as to which mechanism reduces $\left|\mu_{\mathrm{RoCoF} i+}\right|$ and $\left|\mu_{\text {RoCoFi- }}\right|$ more, and, in most cases, it is the INP.

When both mechanisms operate simultaneously, $\left|\mu_{\text {Psci+ }}\right|$ and $\left|\mu_{P s c i-}\right|$ are greatly reduced according to Table 3. However, the results of the separate operation of the mechanisms show that the impact of INP is greater than the impact of CBRR. This is expected, as the CBRR only activates the RRs in the cooperating CAs. Moreover, the reduction of $\left|\sigma_{P s c i+}\right|$ and $\left|\sigma_{P s c i-}\right|$ is most noticeable when both mechanisms operate simultaneously. However, the results of the separate operation of the mechanisms show that the impact of the INP is greater than the impact of the CBRR.

The conclusions for $\left|\Delta W_{\mathrm{e} i+}\right|$ and $\left|\Delta W_{\mathrm{e} i-}\right|$ are similar to $\left|\mu_{P_{\mathrm{sc} i+}}\right|$ and $\left|\mu_{P s c i-}\right|$ according to Table 4, which is expected, as this indicator describes the response of the control units.

When both mechanisms operate simultaneously, $\left|\Delta W_{\mathrm{uni+}}\right|$ and $\left|\Delta W_{\mathrm{uni} i-}\right|$ are reduced according to Table 4 , except in one case where only $\left|\Delta W_{\text {uni+ }}\right|$ was increased, while $\left|\Delta W_{\text {uni- }}\right|$ was reduced considerably. Furthermore, the results of the separate operation of the mechanisms show that the INP almost completely eliminates unintentional deviations, while the impact of the CBRR is not very pronounced.

When both mechanisms operate simultaneously, $\left|W_{\text {cori+ }}^{\mathrm{INP}}\right|,\left|W_{\mathrm{cor} i-}^{\mathrm{INP}}\right|,\left|W_{\mathrm{cori}+}^{\mathrm{CBRR}}\right|$ and $\left|W_{\text {cori- }}^{\mathrm{CBRR}}\right|$ are slightly reduced compared to separate operation of the INP and CBRR according to Table 5 . This is due to the $P_{\text {cori }}$ adjustment mechanism, which is only required in the case of simultaneous operation of the INP and CBRR.

Moreover, simulations of the simultaneous operation of the INP and CBRR were performed for different values of $H_{i}, T_{i j}$, and $R_{i}$. The results are given in Figures 14-16.

There are no unambiguous conclusions about the impact of the mechanisms on $\sigma_{\Delta f i}$, and the differences are extremely small, as shown in Figure 14 (left). In addition, the impact of $H_{i}$ and $T_{i j}$ is not clear, whereas the increase of $R_{i}$ results in an increase of $\sigma_{\Delta f i}$. However, 
both mechanisms significantly reduce $\sigma_{A C E i}$, as shown in Figure 14 (right), whereas $H_{i}, T_{i j}$ and $R_{i}$ have no impact on $\sigma_{A C E i}$.

Table 4. Balancing energy and unintended exchange of energy.

\begin{tabular}{ccccccccc}
\hline \multirow{2}{*}{ Indicator } & \multicolumn{4}{c}{$\Delta \boldsymbol{W}_{\mathbf{e} i+}[\mathbf{p u ~ h}]$} & \multicolumn{5}{c}{$\Delta \boldsymbol{W}_{\mathbf{e} i-}[\mathbf{p u ~ h}]$} \\
\cline { 2 - 9 } & wo & INP/CBRR & INP & CBRR & wo & INP/CBRR & INP & CBRR \\
\hline $\mathrm{CA}_{1}$ & 8.533 & 7.465 & 6.478 & 9.127 & -9.469 & -5.216 & -5.229 & -8.699 \\
$\mathrm{CA}_{2}$ & 16.033 & 8.481 & 10.565 & 12.690 & -4.713 & -3.014 & -3.306 & -3.983 \\
$\mathrm{CA}_{3}$ & 9.785 & 7.567 & 7.603 & 9.224 & -10.059 & -5.172 & -6.000 & -8.518 \\
\hline \multirow{2}{*}{ indicator } & \multicolumn{4}{c}{$\Delta W_{\text {uni+ }}[\mathrm{pu} \mathrm{h}]$} \\
\cline { 2 - 9 } & wo & INP/CBRR & INP & CBRR & wo & INP/CBRR & INP & CBRR \\
\hline $\mathrm{CA}_{1}$ & 7.750 & 5.937 & 0.628 & 6.769 & -4.531 & -3.551 & -0.607 & -4.023 \\
$\mathrm{CA}_{2}$ & 3.916 & 5.431 & 0.721 & 6.358 & -9.803 & -2.745 & -0.733 & -2.857 \\
$\mathrm{CA}_{3}$ & 7.462 & 5.771 & 0.611 & 6.302 & -4.794 & -3.218 & -0.620 & -3.444 \\
\hline \hline
\end{tabular}

Legend: wo-without the INP/CBRR, INP/CBRR—simultaneous operation, INP—separate operation, CBRRseparate operation.

Table 5. Energy exchange.

\begin{tabular}{|c|c|c|c|c|c|c|}
\hline \multirow{2}{*}{ Indicator } & \multicolumn{3}{|c|}{$W_{\text {cori+ }}^{\mathrm{INP}}[\mathrm{pu} \mathrm{h}]$} & \multicolumn{3}{|c|}{$W_{\text {cori- }}^{\text {INP }}[\mathrm{pu} \mathrm{h}$ ] } \\
\hline & INP/CBRR & INP & CBRR & INP/CBRR & INP & CBRR \\
\hline $\mathrm{CA}_{1}$ & 5.147 & 5.165 & 0 & -2.965 & -2.976 & 0 \\
\hline $\mathrm{CA}_{2}$ & 2.012 & 2.016 & 0 & -6.068 & -6.083 & 0 \\
\hline $\mathrm{CA}_{3}$ & 4.824 & 4.840 & 0 & -2.950 & -2.961 & 0 \\
\hline \multirow{2}{*}{ indicator } & \multicolumn{3}{|c|}{$W_{\text {cori+ }}^{\text {CBRR }}$ [pu h] } & \multicolumn{3}{|c|}{$W_{\text {cori- }}^{\text {CBRR }}[\mathrm{pu} \mathrm{h]}$} \\
\hline & INP/CBRR & INP & CBRR & INP/CBRR & INP & CBRR \\
\hline $\mathrm{CA}_{1}$ & 3.472 & 0 & 4.047 & -4.831 & 0 & -5.405 \\
\hline $\mathrm{CA}_{2}$ & 2.783 & 0 & 2.884 & -7.285 & 0 & -8.745 \\
\hline $\mathrm{CA}_{3}$ & 3.844 & 0 & 4.590 & -5.610 & 0 & -6.475 \\
\hline
\end{tabular}

Legend: INP/CBRR—simultaneous operation, INP—separate operation, CBRR—separate operation.

Generally, both mechanisms reduce the $\left|\mu_{\text {RoCoFi+ }}\right|$ and $\left|\mu_{\text {RoCoFi- }}\right|$, as shown in Figure 15. In addition, an increase of $H_{i}$ and $R_{i}$ results in a decrease of $\left|\mu_{\text {RoCoFi+ }}\right|$ and $\left|\mu_{\text {RoCoFi- }}\right|$, whereas an increase of $T_{i j}$ results in an increase of $\left|\mu_{\mathrm{RoCoFi+}}\right|$ and $\left|\mu_{\mathrm{RoCOFi-}}\right|$. Note that, when $T_{i j}=1 / 15 \mathrm{pu} / \mathrm{Hz},\left|\mu_{\mathrm{RoCoFi}+}\right|$ and $\left|\mu_{\mathrm{RoCoFi}}\right|$ is increased with the mechanisms.

When both mechanisms operate simultaneously, $\left|\mu_{\mathrm{Psc}_{+}}\right|$and $\left|\mu_{\mathrm{Psci}-}\right|$ are greatly reduced, as shown in Figure 16. However, $H_{i}, T_{i j}$ and $R_{i}$ have no impact on $\left|\mu_{\text {Psci }}\right|$ and $\left|\mu_{P s c i-}\right|$.
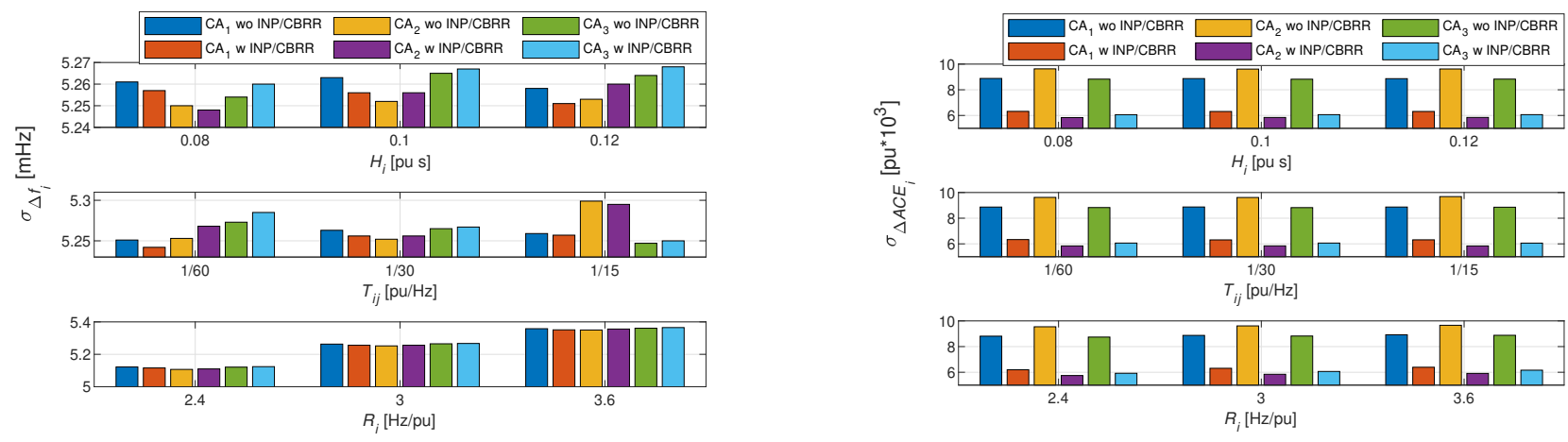

Figure 14. Average values of $\sigma_{\Delta f_{i}}$ (left) and average values of $\sigma_{A C E_{i}}$ (right) for different values of $H_{i}, T_{i j}$ and $R_{i}$, where "wo INP/CBRR" is without and "w INP/CBRR" is with the INP and CBRR. 

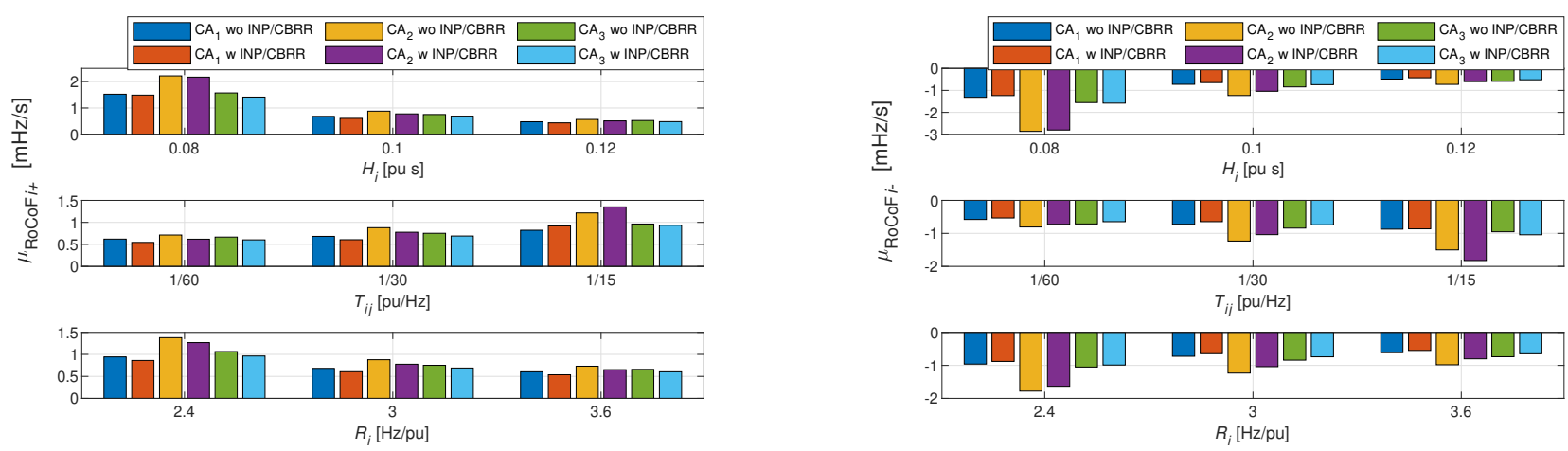

Figure 15. Average values of $\mu_{\mathrm{RoCoF}_{i+}}$ (left) and average values of $\mu_{\mathrm{RoCoF}_{i-}}$ (right) for different values of $H_{i}, T_{i j}$ and $R_{i}$, where "wo INP/CBRR" is without and "w INP/CBRR" is with the INP and CBRR.
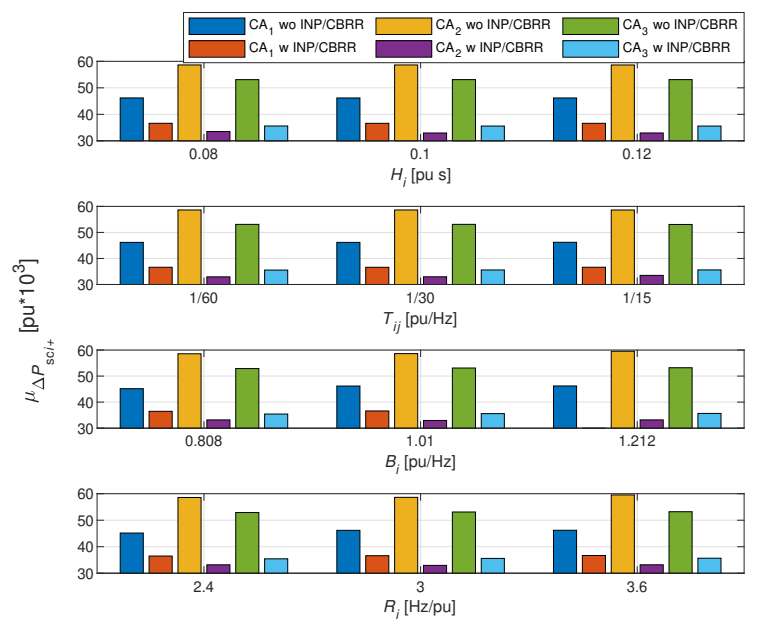
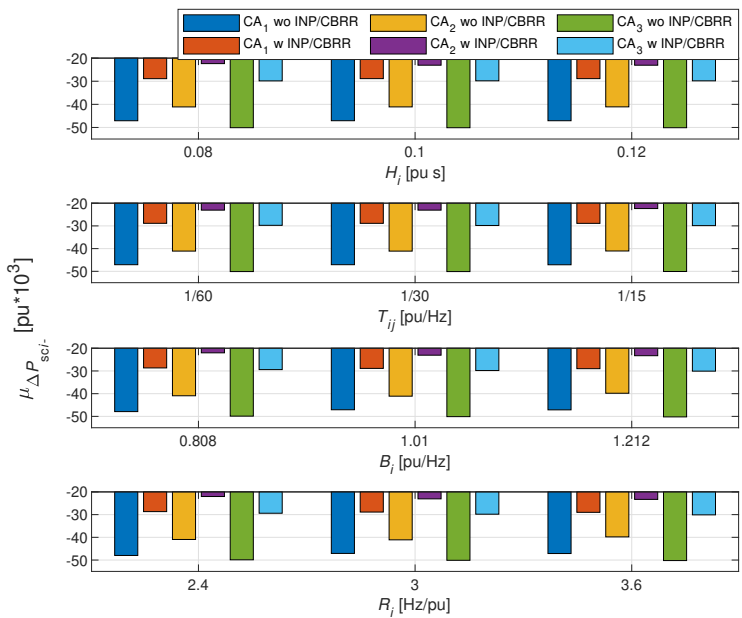

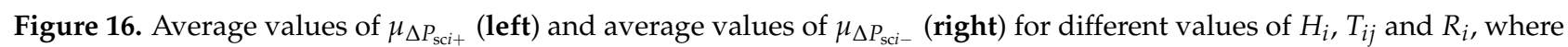
"wo INP/CBRR" is without and "w INP/CBRR" is with the INP and CBRR.

\section{Conclusions}

This article discusses the simultaneous operation of the INP and CBRR, which no previous studies have investigated. Extensive dynamic simulations of a three-CA test system with the simultaneous operation of the INP and CBRR were performed to evaluate their impact on the frequency quality, the LFC, and performance.

The results confirmed the conclusions in [20-23], where the INP and CBRR were analyzed separately. The results of the step change of load and the random load variation confirmed that the impact of the INP and CBRR on frequency deviations has no unambiguous conclusions. In addition, the impact of inertia and synchronizing coefficient is not clear, whereas the increase of droop characteristic results in an increase of frequency deviations. However, both mechanisms reduce the ACE deviations-the INP slightly more than CBRR. The reduction is most pronounced when both mechanisms operate simultaneously. Moreover, the function for correction power adjustment additionally decreased ACE and scheduled control power, which prevents undesirable switching between the INP and CBRR. Both mechanisms also reduce the RoCoF, and the reduction is most pronounced when both mechanisms operate simultaneously. However, there are no unambiguous conclusions as to which mechanism reduces the RoCoF more, and, in most cases, it is the INP. Increase of inertia and droop characteristic results in a decrease of RoCoF, whereas increase of synchronizing coefficients results in an increase of RoCoF. When both mechanisms operate simultaneously, the scheduled control power is greatly reduced and the impact of the INP is greater than the impact of the CBRR. Similarly, the balancing energy as well as the unintended exchange of energy are greatly reduced when both mechanisms operate simultaneously. However, there is no impact of inertia, synchronizing coefficient, and droop characteristic on scheduled control power. Due to the function for correction 
power adjustment, which prevents undesirable activation of the INP and CBRR, energy exchange was slightly reduced, as expected. Because of the reduced unintended exchange of energy, beneficial economic consequences can be anticipated when the INP and CBRR operate simultaneously.

One of the tough challenges for all researchers in this domain is the dynamic dimensioning of RRs, considering the INP and CBRR. This article clearly shows that the simultaneous operation of the INP and CBRR reduces the activation of the RRs, which is currently not considered in the reserve dimensioning process.

Author Contributions: Supervision, B.P.; Writing—original draft, M.T.; Writing—review and editing, B.P. All authors have read and agreed to the published version of the manuscript.

Funding: This work was supported by ARRS under Projects P2-0115 and L2-7556.

Institutional Review Board Statement: Not applicable.

Informed Consent Statement: Not applicable.

Data Availability Statement: This study did not report any data.

Conflicts of Interest: The authors declare no conflict of interest.

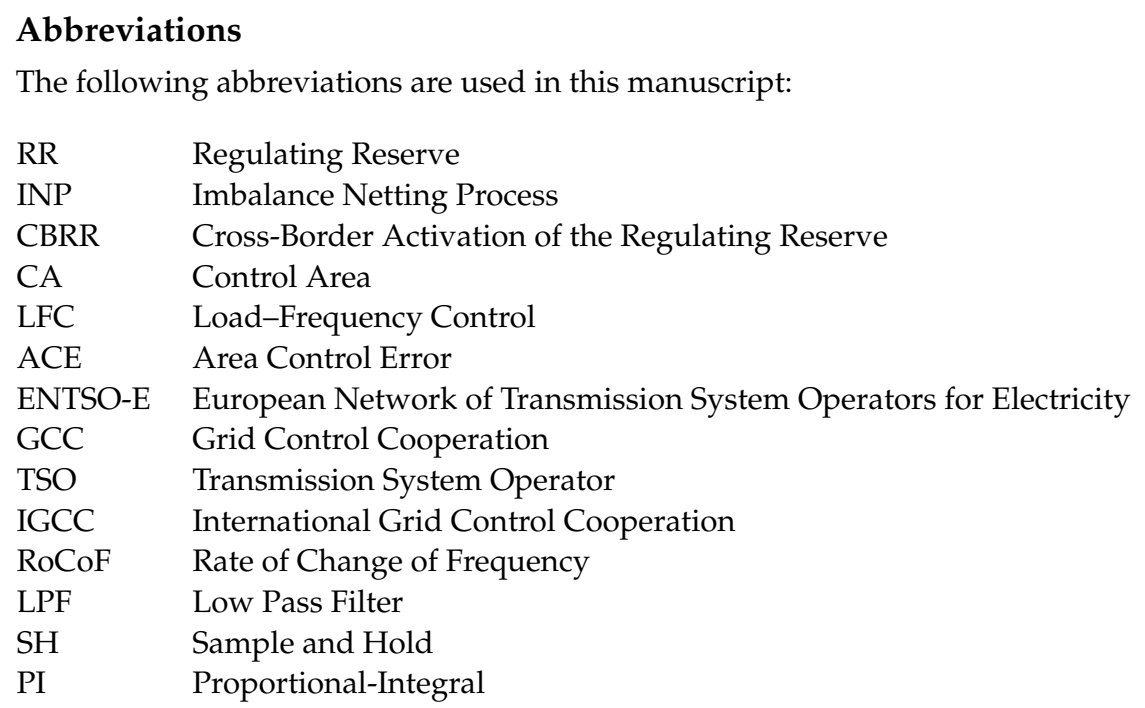

\section{References}

1. EUR-Lex. Establishing a Guideline on Electricity Transmission System Operation. Commission Regulation (EU) $2017 / 1485$. Available online: https:/ / eur-lex.europa.eu/eli/reg/2017/1485/oj (accessed on 15 July 2021).

2. EUR-Lex. Establishing a Guideline on Electricity Balancing. Commission Regulation (EU) 2017/2195. Available online: https: / / eur-lex.europa.eu/eli/reg/2017/2195/oj (accessed on 15 July 2021).

3. ENTSO-E. Proposal for the Implementation Framework for a European Platform for the Exchange of Balancing Energy from Frequency Restoration Reserves; ENTSO-E: Brussels, Belgium, 2020.

4. ENTSO-E. IGCC Regular Report on Social Welfare; ENTSO-E: Brussels, Belgium, 2020.

5. Zolotarev, P.; Gökeler, M.; Kuring, M.; Neumann, H.; Kurscheid, E.M. Grid Control Cooperation-A Framework for Technical and Economical Crossborder Optimization for Load-Frequency Control. In Proceedings of the 44th International Conference on Large High Voltage Electric Systems (Cigre'12), Paris, France, 26-30 August 2012.

6. Pawalek, A.; Hofmann, L. Utilization of HVDC-Systems in the International Grid Control Cooperation. In Proceedings of the IEEE Milan PowerTech 2019, Milan, Italy, 23-27 June 2019.

7. Pawalek, A.; Hofmann, L. Comparison of Methods for the Simulation of Dynamic Power Flows in the International Grid Control Cooperation. In Proceedings of the IEEE Electronic Power Grid (eGrid) 2018, Charleston, SC, USA, 12-14 November 2018.

8. Cronenberg, A; Sager, N.; Willemsen, S. Integration of France Into the International Grid Control Cooperation. In Proceedings of the IEEE International Energy Conference 2016 (ENERGYCON'16), Leuven, Belgium, 4-8 April 2016.

9. ENTSO-E. Stakeholder Document for the Principles of IGCC; ENTSO-E: Brussels, Belgium, 2020.

10. ENTSO-E. Supporting Document for the Network Code on Load-Frequency Control and Reserves; ENTSO-E: Brussels, Belgium, 2013. 
11. Zolotarev, P. Social Welfare of Balancing Markets. In Proceedings of the 14th International Conference on the European Energy Market (EEM) 2017, Dresden, Germany, 6-9 June 2017.

12. Avramiotis, F.I.; Margelou, S.; Zima, M. Investigations on a Fair TSO-TSO Settlement for the Imbalance Netting Process in European Power System. In Proceedings of the 15th International Conference on the European Energy Market (EEM) 2018, Lodz, Poland, 27-29 June 2018.

13. Oneal, A.R. A Simple Method for Improving Control Area Performance: Area Control Error (ACE) Diversity Interchange ADI. IEEE Trans. Power Syst. 1995, 10, 1071-1076. [CrossRef]

14. Zhou, N.; Etingov, P.V.; Makarov, J.V.; Guttromson, R.T.; McManus, B. Improving Area Control Error Diversity Interchange (ADI) Program by Incorporating Congestion Constraints. In Proceedings of the IEEE PES T\&D 2010, New Orleans, LA, USA, 19-22 April 2010.

15. Apostolopoulou, D.; Sauer, P.W.; Dominguez-Garcia, A.D. Balancing Authority Area Coordination With Limited Exchange of Information. In Proceedings of the IEEE Power and Energy Society General Meeting 2015, Denver, CO, USA, 26-30 July 2015.

16. ENTSO-E. Anual Report 2019 Edition; ENTSO-E: Brussels, Belgium, 2020.

17. EXPLORE. Target Model For Exchange of Frequency Restoration Reserves. Available online: https://www.tennet.eu/news/ detail/explore-target-model-for-exchange-of-frequency-restoration-reserves/ (accessed on 15 July 2021).

18. ENTSO-E. Consultation on the Design of the Platform for Automatic Frequency Restoration Reserve (AFRR) of PICASSO Region; ENTSO-E: Brussels, Belgium, 2012.

19. Gebrekiros, T.Y. Analysis of Integrated Balancing Markets in Northern Europe under Different Market Design Options; NTNU: Trondheim, Norway, 2015.

20. Topler, M.; Polajžer, B. Impact of Imbalance Netting Cooperation on Frequency Quality and Provision of Load-Frequency Control. In Proceedings of the IEEE International Conference on Environment and Electrical Engineering 2019 and IEEE Industrial and Commercial Power Systems Europe (EEEIC/I\&CPS Europe) 2019, Genoa, Italy, 11-14 June 2019.

21. Topler, M.; Ritonja, J.; Polajžer, B. The Impact of the Imbalance Netting Process on Power System Dynamics. Energies 2019, 12, 1-18. [CrossRef]

22. Topler, M.; Polajžer, B. Evaluation of the Cross-Border Activation of the Regulating Reserve with Dynamic Simulations. In Proceedings of the IEEE International Conference on Environment and Electrical Engineering 2020 and IEEE Industrial and Commercial Power Systems Europe (EEEIC/I\&CPS Europe) 2020, Madrid, Spain, 9-12 June 2020.

23. Topler, M.; Ritonja, J.; Polajžer, B. Improving the Cross-Border Activation of the Regulating Reserve to Enhance the Provision of Load-Frequency Control. IEEE Access 2020, 8, 170696-170712. [CrossRef]

24. Lu, C.W.; Lin, G.H.; Wu, T.J.; Hu, I.H.; Chang, Y.C. Influencing Factors of Cross-Border E-Commerce Consumer Purchase Intention Based on Wireless Network and Machine Learning. Secur. Commun. Netw. 2021, 2021, 9984213. [CrossRef]

25. Imani, M.; Ghoreishi, S.F. Graph-Based Bayesian Optimization for Large-Scale Objective-Based Experimental Design. IEEE Trans. Neural Netw. Learn. Syst. 2021, 1-13. [CrossRef]

26. Meltzer, J.P.; Lovelock, P. Regulating for a Digital Economy-Understanding the Importance of Cross-Border Data Flows in Asia. Glob. Econ. Dev. Work. Pap. 2018, 113, 1-51.

27. ENTSO-E. Operational Reserve Ad Hoc Team Report; ENTSO-E: Brussels, Belgium, 2012.

28. Bevrani, H. Robust Power System Frequency Control; Springer: New York, NY, USA, 2009.

29. Kundur, P. Power System Stability and Control; McGraw-Hill: New York, NY, USA, 1994.

30. NERC. Frequency Response Standard, Background Document; NERC: Atlanta, GA, USA, 2012.

31. ENTSO-E. Rate of Change of Frequency (ROCOF) Withstand Capability; ENTSO-E: Brussels, Belgium, 2017.

32. ACER. Benefits from Balancing Market Integration (Imbalance Netting and Exchange of Balancing Energy); ACER: Ljubljana, Slovenia, 2018.

33. Anderson, P.M.; Mirheydar, M. A Low-Order System Frequency Response Model. IEEE Trans. Power Syst. 1990, 5, 720-729. [CrossRef]

34. Elgerd, O.I.; Fosha, C.E. Optimum Mmegawatt-Frequency Control of Multiarea Electric Energy Systems. IEEE Trans. Power App. Syst. 1970, 89, 556-563. [CrossRef]

35. Vorobev, P.; Greenwood, D.M.; Bell, J.H; Bialek, J.W.; Taylor, P.C.; Turitsyn, K. Deadbands, Droop, and Inertia Impact on Power System Frequency Distribution. IEEE Trans. Power Syst. 2019, 34, 3098-3108. [CrossRef]

36. Kundur, P. Definition and Classification of Power System Stability IEEE/CIGRE Joint Task Force on Stability Terms and Definitions. IEEE Trans. Power Syst. 2004, 19, 1387-1401.

37. Janecek, E.; Cerny, V.; Fialova, A.; Fantík, J. A New Approach to Modelling of Electricity Transmission System Operation. In Proceedings of the IEEE PES Power Systems Conference and Exposition 2006, Atlanta, GA, USA, 29 October-1 November 2006. 